\title{
Sit-to-stand-Tests sind aussagekräftig als Fitness-Check bei COPD
}

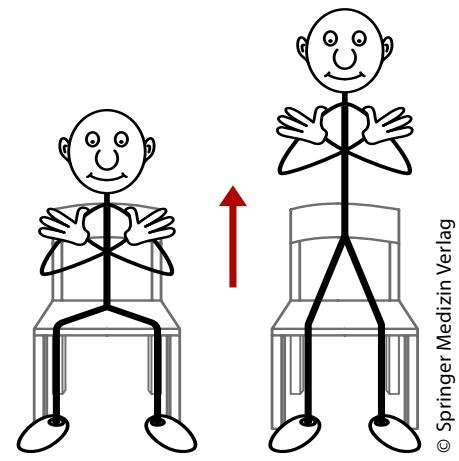

Hintergrund u. Fragestellung: Sit-to-stand Tests (STST, s. $\triangleright$ Abb.) wurden als einfache funktionelle Tests entwickelt, um die körperliche Leistungsfähigkeit u.a. bei Patienten mit COPD zu prüfen. Es bestehen verschiedene Durchführungsformen dieser Tests. Das vorliegende Review gibt einen Überblick über die Vorteile und Limitationen dieser Messverfahren mit dem Ziel, für spezifische Fragestellungen die optimale Variante zu wählen.

Patienten u. Methoden: 17 Originalarbeiten, die sich mit Originalie

Vaidya T, Chambellan A, de Bisschop C. Sit-to-stand tests for COPD: A literature review. Respir Med. 2017 Jul;128:70-77 kunden bis 3 Minuten) prüften. Die jeweiligen Ergebnisse der STSTs wurden mit denen weiterer funktioneller Tests verglichen.

Ergebnisse: Eine Vielzahl hoher Korrelationen wurde erreicht zwischen der STST-Leistung und den Ergebnissen anderer funktioneller Tests (z.B. Korrelation 6-Minuten Gehteststrecke versus geschaffte Wiederholungen beim 1-Min STST: $r=0,75$, $\mathrm{p}<0,001)$. STSTs sind einfach, mit geringem räumlichem Bedarf durchzuführen und haben eine hervorragende Validität und Sensitivität (z.B. auf Veränderungen nach Reha).

Schlussfolgerungen: STSTs scheinen ein valides und relevantes Messinstrument zu sein, um die funktionelle Leistungsfähigkeit bei COPD-Patienten zu beurteilen. Dennoch müssen verschiedene Varianten der STSTs bei COPD-Patienten unterschiedlich bewertet und interpretiert werden.

\section{- Kommentar von Dr. phil. Rainer Glöckl}

\section{Geringer Aufwand und Platzbedarf von Vorteil}

Die erste wissenschaftliche Erwähnung eines STSTs tauchte im Jahr 1985 auf. Ziel war es, anhand einer funktionellen Alltagsbewegung (Aufstehen und Hinsetzen) Anhaltspunkte für die Beinkraft von Menschen verschiedener Altersklassen zu erhalten. Im Lauf der Jahre entwickelten sich verschiedene Formen, welche bei unterschiedlichen Populationen getestet wurden, um u.a. Rückschlüsse auf die Balancefähigkeit oder das potentielle Sturzrisiko zu ziehen. Bei pneumologischen Patienten wurden STSTs erst in der jüngeren Vergangenheit und einer kleinen Anzahl von Studien ( $n=17)$ untersucht.

Im Grunde haben sich vor allem 2 Varianten von STSTs etabliert. Zum einen der 5-Wiederholungs-STST und der 1-MinutenSTST. Beide korrelieren signifikant mit der Beinkraft und der 6-Minuten-Gehteststrecke. Dennoch gibt es deutliche Unterschiede: Der 5-Wiederholungen-STST testet vor allem die Aspekte der Kraftfähigkeit und Koordination - der 1-Min.-STST eher die Kraftausdauer und die allgemeine körperliche Leistungsfähigkeit. Für beide Testvarianten wurde bereits definiert, welche Veränderungen bei den Tests als klinisch relevant interpretiert werden können. Beim 5-Wiederholungen-STST kann eine klinisch relevante Verbesserung ab einer Reduktion der Testdauer um mindestens 1,7 Sekunden - beim 1-Min.-STST ab einer Steigerung um 3 Wiederholungen angenommen werden. Der 1-Min.-STST kann darüber hinaus Aufschlüsse über die Prognose von COPD-Patienten geben. Können Patienten weniger als 12 Wiederholungen durchführen, so haben sie in den nächsten 2 Jahren ein signifikant erhöhtes Mortalitätsrisiko. Patienten, die mehr als 20 Aufstehbewegungen in einer Minute absolvieren können, haben eine deutlich positivere Prognose.
Nachteile von STSTs sind, dass diese von körperlich sehr schwachen Patienten, die nicht in der Lage sind, ohne fremde Hilfe aufzustehen, nicht durchgeführt werden können. Zudem ist der 5-Wiederholungen-STST bei körperlich sehr fitten Patienten aufgrund eines gewissen Plateau-Effektes nicht mehr aussagekräftig, da der Test nicht beliebig schneller durchgeführt werden kann.

Die Vorteile von STSTs sind dennoch überzeugend: STSTs sind schnell absolviert, sie benötigen kein teures Equipment (nur Stuhl und Stoppuhr), sie unterliegen so gut wie keinem Lerneffekt (Intraklassen-Korrelationskoeffizient: $>0,93$ ), sie testen die alltagsnahe funktionelle Leistungsfähigkeit, liefern eine klinisch relevante Aussage und benötigen kaum Platz. Vor allem aufgrund des Platzaspektes können STSTs als sinnvolle Belastungstests für Institutionen v.a. auch mit räumlich begrenzten Möglichkeiten (z.B. Arzt- oder Physiotherapiepraxen) angesehen werden.

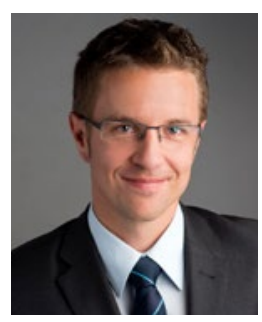

Dr. phil. Rainer Glöckl

Fachzentrum Pneumologie

Schön Klinik Berchtesgadener Land

Malterhöh 1

83471 Schönau am Königssee

rgloeckl@schoen-kliniken.de 